Revista

Ibero-Americana

de Estratégıa

\title{
VALOR DA EMPRESA E MÉTRICAS DE MARKETING
}

\author{
VALUE OF THE COMPANY AND MARKETING METRICS
}

\section{VALOR DE LA EMPRESA Y MARKETING MÉTRICAS}

\section{André Luiz Ramos}

Doutorando em Administração pela Universidade Nove de Julho - UNINOVE

Professor da Universidade Nove de Julho - UNINOVE

E-mail: andre.ramos@uninove.br (Brasil)

\section{Otávio Bandeira De Lamônica Freire}

Doutor em Ciências da Comunicação pela Universidade de São Paulo - USP

Professor do Programa de Pós-Graduação em Administração da Universidade Nove de Julho PPGA/UNINOVE

E-mail: otaviofreire@uninove.br (Brasil)

\section{Marcelo Moll Brandão}

Doutor em Administração de Empresas pela Fundação Getulio Vargas - FGV

Professor do Programa de Pós-Graduação em Administração da Universidade Nove de Julho PPGA/UNINOVE

E-mail: marcelo.brandao@uninove.br (Brasil) 


\section{VALOR DA EMPRESA E MÉTRICAS DE MARKETING}

\section{RESUMO}

A partir do delineamento das estratégias de marketing na perspectiva da visão baseada em recursos (Barney, 1991), onde os ativos podem ser físicos, organizacionais e humanos, e Constantin e Lusch (1994), que tratam os recursos estratégicos como recursos tangíveis ou intangíveis, internos ou externos à firma, surge uma linha de pesquisa de marketing e finanças. Segundo Srivastava, Shervani e Fahey (1998) são três os tipos de ativo de mercado que geram resultados no valor da empresa. O valor de uma empresa pode ser medido pelo fluxo de caixa descontado, comprometendo as atividades de marketing à previsão de geração de valor (Anderson, 1982; Day, Fahey, 1988; Doyle, 2000; Rust et al., 2004a). O valor econômico das estratégias e das métricas de marketing recebe interesse crescente dos pesquisadores de estratégia e dos gestores de marketing. É cada vez mais latente a necessidade de uma ponte que articule marketing e finanças em uma perspectiva estratégica. Este artigo se propõe um framework analítico, a partir das diferentes abordagens científicas envolvendo risco e retorno promovido pelas estratégias de marketing e aponta os avanços concernentes tanto às abordagens metodológicas, quanto às estratégias de marketing e seu impacto nas métricas e valor da firma a partir do estudo de Srinivasan e Hanssens (2009).

\section{VALUE OF THE COMPANY AND MARKETING METRICS}

\section{ABSTRACT}

Thinking marketing strategies from a resource-based perspective (Barney, 1991), proposing assets as either tangible, organizational and human, and from Constantin and Luch's vision (1994), where strategic resources can be tanbigle or intangible, internal or external to the firm, raises a research approach on Marketing and Finance. According to Srivastava, Shervani and Fahey (1998) there are 3 market assets types, which generate firm value. Firm value can be measured by discounted cashflow, compromising marketing activities with value generation forcasts (Anderson, 1982; Day, Fahey, 1988; Doyle, 2000; Rust et al., 2004a). The economic value of marketing strategies and marketing metrics are calling strategy researchers' and marketing managers' attention, making clear the need for building a bridge able to articulate marketing and finance form a strategic perspective. This article proposes an analytical framework based on different scientific approaches envolving risk and return promoted by marketing strategies and points out advances concerning both methodological approaches and marketing strategies and its impact on firm metrics and value, usgin Srinivasan and Hanssens (2009) as a start point. 


\section{VALOR DE LA EMPRESA Y MARKETING MÉTRICAS}

\section{RESUMEN}

Desde el diseño de estrategias de marketing desde la perspectiva de la basada en los recursos ( Barney, 1991 ), donde los activos pueden ser Constantin y Lusch ( 1994 ) vista físico, organizativo y humano, y, que trata de recursos estratégicos como activos tangibles o intangibles internos o externos a la empresa, una línea de marketing en buscadores y finanzas surge . Según Srivastava, Shervani y Fahey ( 1998 ) existen tres tipos de activos de los mercados que generan resultados en valor de la empresa. El valor de una empresa se puede medir por el flujo de caja descontado, que afecta a las actividades de marketing de la generación de valor pronóstico (Anderson, 1982 ; Día, Fahey, 1988 ; Doyle, 2000 ; . Rust et al, 2004a ). El valor económico de las estrategias y las métricas de marketing recibe creciente interés por los investigadores en la estrategia de marketing y gerentes. Es necesidad cada vez más latente de un puente que une el marketing y las finanzas desde una perspectiva estratégica. En este artículo se propone un marco de análisis, desde diferentes enfoques científicos que implica riesgo y rendimiento impulsado por las estrategias de marketing y los puntos pueden estar relacionados con los avances metodológicos ambos enfoques con respecto a las estrategias de marketing y su impacto en los indicadores de valor y firme por parte de estudiar Srinivasan y Hanssens ( 2009 ). 


\section{INTRODUÇÃO}

A discussão sobre marketing e finanças ganhou espaço na academia nos últimos 20 anos, a partir de Day e Fahey (1988) que relacionaram estratégias de mercado e criação de valor da firma, enfatizando as estratégias empresariais em detrimento de projetos individuais, evidenciado maior retorno para empresas que pensavam e planejavam suas estratégias de mercado de forma alinhada e no longo prazo. Com base na perspectiva de ações baseadas no mercado - introduzida por Sharp (1995) - Srivastava, Shervani e Fahey (1998) apresentaram as bases conceituais para o entendimento das ações de marketing como ativos estratégicos de mercado que se relacionam com o valor da empresa por meio do fluxo de caixa.

A partir do delineamento das ações de marketing na perspectiva da visão baseada em recursos (Barney, 1991), que propõe que os ativos podem ser físicos, organizacionais e humanos, e Constantin e Lusch (1994), que tratam os recursos estratégicos como recursos tangíveis ou intangíveis, internos ou externos à firma, surge uma linha de pesquisa de marketing e finanças. Segundo Srivastava, Shervani e Fahey (1998) são três os tipos de ativo de mercado que geram resultados diretos ou indiretos no valor da empresa - ativos de mercado baseados na relação entre a empresa e seus stakeholders principais; ativos baseados no conhecimento da empresa em relação aos clientes, concorrentes e canais; e o valor da marca e a soma de valor que cada ativo influencia positivamente ou negativamente para o valor da marca. Conforme estudos relevantes na área de finanças e marketing, recursos estratégicos desenvolvidos por meio de atividades e estratégias de marketing serão tratados por "ativos de mercado", "ativos de marketing", ou "estratégias de marketing"

O valor de uma empresa pode ser medido pelo fluxo de caixa descontado, o que faz com que atividades de marketing estejam comprometidas com a previsão de geração de valor para a empresa (Anderson, 1982; Srivastava, Shervani, Fahey, 1998; Day, Fahey, 1988; Doyle, 2000; Rust et al., 2004a). Promoções de preço antecipam vendas, estratégias de relacionamento diminuem a sua volatilidade, novos produtos e extensões de marca podem gerar novas ondas positivas de receita nos fluxos já existentes (Srivastava, Shervani, Fahey, 1998).

O valor econômico das decisões estratégicas de marketing vem recebendo interesse crescente na literatura de marketing (Hyman, Mathur, 2005; Greenyer, 2006; Kimbrough, McAlister, 2009). Estudos examinam os efeitos de decisões estratégicas de marketing - inovações de produto, gastos com pesquisa e desenvolvimento, despesas de publicidade, qualidade do produto e satisfação do consumidor - na rentabilidade da empresa e no valor de suas ações (Kaldor, 1950; Kirmani, Wright, 1989; Capon, Farley, Hoenig, 1990; Erickson, Jacobson, 1992; Szymanski, 
Bharadwaj, Varadarajan, 1993; Leone, 1995; Knowles, 2003; Lehman, 2004; Byzalov, Shachar, 2004; Grullon, Kanatas, Weston, 2004; Fehle, Tsyplakov, Zdorovtsov, 2005; Chemmanur, Yan, 2009a, 2009b; Luo, Homburg, Wieseke, 2010).

Nesse sentido, estudos posteriores aos artigos seminais de finanças e marketing tratam a mensuração das estratégias de marketing como métricas de marketing. Em geral, as métricas de marketing se propõem a mensurar em que medida diferentes ações e estratégias de marketing influenciam o valor da empresa (Luo, Homburg, Wieseke, 2010).

Métricas de marketing têm recebido atenção considerável de gestores e acadêmicos nos últimos anos. Do ponto de vista gerencial, a alta administração pede cada vez mais posicionamento da área, no que diz respeito a métodos para se avaliar o desempenho de suas ações (Ambler, 2003; CMO Council, 2004; Rust et al., 2004a, 2004b; Sheth, Sisodia, 2001; Woodburn, 2004; Xueming, Naveen, 2006; Xueming, Jong, 2012).

Diante deste contexto, é cada vez mais latente a necessidade de uma ponte empírica que articule marketing e finanças, procurando apresentar soluções para as diversas questões que concernem a contribuição financeira das estratégias de marketing para o valor gerado para os acionistas (Day, Fahey, 1988; Doyle, 2000; Srivastava, Shervani, Fahey, 1998; Lehman, 2004; Hyman, Mathur, 2005; Luo, Homburg, Wieseke, 2010).

Apesar de uma iniciativa de marketing levar a vários resultados significativos (por exemplo, a satisfação do cliente, a lealdade, a quota de mercado), o reconhecimento desta iniciativa ocorre somente se estes resultados também, em última análise, indicarem um aumento na riqueza dos acionistas (Ramesh, Bharadwaj, 2008).

Srinivasan e Hanssens (2009), em um artigo relevante na área de estratégias de marketing e finanças, propõem um framework analítico, a partir das diferentes abordagens científicas envolvendo risco e retorno promovido pelas estratégias de marketing. Assim sendo, o presente artigo propõe o apontamento dos avanços concernentes tanto às abordagens metodológicas, quanto às estratégias de marketing e seu impacto nas métricas e valor da firma a partir do estudo de Srinivasan e Hanssens (2009).

O estudo será essencialmente bibliográfico e pretende-se, a partir do levantamento teórico, contribuir com um esquema teórico e proposições teóricas que possam ser testadas empiricamente em estudos futuros.

A relevância deste trabalho reside no fato de haver poucos estudos relacionando ações de marketing como recurso estratégico e métricas e valor da empresa. Além disso, poucos são os estudos encontrados no Brasil, em sua maioria, trabalhos publicados somente em congressos da área 
(Estellita, Carvalho, Lima, 2006; Brandão et al. 2006; Fortunato, Ness, Motta, 2009; Martins, 2011; Freundt, 2012).

Soma-se à falta de estudos sobre finanças e marketing no contexto brasileiro a entrada de novos investidores no mercado de ações, principalmente no que tange aos pequenos investidores. Esses novos participantes do mercado de ações não são especialistas e podem ser mais positivamente influenciados pelas ações de marketing das empresas, mesmo quando essas não gerem valor para o negócio. Os estudos sobre a relação entre métricas de marketing e valor da firma devem incrementar o conhecimento sobre estratégias de marketing e retorno tanto para a academia como para os investidores.

\section{FUNDAMENTAÇÃO TEÓRICA}

\subsection{ESTRATÉGIAS DE MARKETING, RISCO E RETORNO DA FIRMA}

Um grande número de estudos tem evidenciado os efeitos das decisões estratégicas de marketing, seja em inovação, pesquisas e desenvolvimento, qualidade de produtos e satisfação de cliente na rentabilidade da empresa (Capon, Farley, Hoenig, 1990; Szymanski, Bharadwaj, Varadarajan,1993; Kremer et al., 2008; Manchanda et al., 2005; Wosinska, 2005; Hyman, Mathur, 2005;).

Srinivasan e Hanssens (2009) sistematizaram o conjunto dos estudos que relacionaram estratégias de marketing e finanças de 1987 a 2009. Em seu framework, existe relação entre o conjunto das estratégias de marketing e os valores da firma. Nesse sentido, o entendimento da relação das estratégias de marketing com os retornos esperados ou anormais passa necessariamente pela análise dos riscos sistemáticos e assistemáticos.

Os estudos que relacionam marketing e finanças podem ser subdivididos em pelo menos cinco perspectivas - four-factor model, event study, calendar portfólio, stock return response model, persistence modeling (Srinivasan e Hanssens, 2009). Independente da perspectiva discutida, as ações gerenciais da empresa em relação ao mercado (ex: para consumidores ou contra concorrentes) atuam como sinal para o mercado e, se forem efetivas, geram resultados diretos em resultados não financeiros (ex: satisfação ou reputação da marca) e ainda os resultados econômicos (ex: valor das ações). Em última instância, os resultados esperados e anormais das empresas serão influenciados pelas ações estratégicas de marketing. 
Para entender as diferentes perspectivas e as variáveis envolvidas, cada uma das perspectivas são explicadas a seguir.

\subsubsection{FOUR-FACTOR MODEL}

Fama e French mostraram que o Capital Asset Pricing Model (CAPM) não era suficiente para explicar os retornos dos ativos nos EUA. A análise de Fama e French (1992, 1993), resultou num modelo alternativo para explicar o retorno das ações, o qual se baseava em três fatores: além do fator de mercado, como encontrado no CAPM, incluíram a relação valor contábil/preço das empresas (valor de mercado da empresa) e o tamanho da empresa. Fama e French (1992, 1993, 1995, 1996, 1998) mostraram documentaram a boa aderência do seu modelo em explicar os retornos das ações, não só os EUA, mas também internacionalmente (Rao, Agarwal, Dahlhoff, 2004; Barth et al., 1998; Madden, Fehle, Fournier, 2006; Chaney et al., 1991; Lane, Jacobson, 1995; Srinivasan e Hanssens, 2009; McAlister et al., 2007). Pesquisas relacionadas sobre o tema no Brasil foram realizadas por Hazzan (1991), Braga, Costa Jr. e Mescolin (1997), Costa Jr. e Neves (2000), Braga e Leal (2000), Lima Jr. (2003) e Cardoso (2006).

O modelo reconhece fontes sistemáticas de diferenças transversais entre as empresas, como o tamanho da empresa, a importância e o valor dos seus ativos intangíveis, a relação do valor de mercado da empresa com o seu valor contábil, a sua classe de risco e a sua dinâmica. Tem como base a Hipótese do Mercado Eficiente (HME), sendo simples de estimar. Como limitação apresenta a sensibilidade à escolha das carteiras de referência, o fato de ser correlacional e a sua vulnerabilidade a vieses devido a omissão de variáveis.

\subsubsection{STOCK RETURN RESPONSE MODEL}

O tempo de abordagem de carteira calendário ou abordagem alfa de Jensen, é uma técnica para analisar o desempenho ajustado ao risco dos investidores privados, empresas ou fundos de investimento por meio de um procedimento em duas etapas.

Desde o trabalho seminal de Jaffe (1974) e Mandelker (1974), esta abordagem tem sido utilizada em diversas áreas empíricas das finanças, como, por exemplo, em pesquisa sobre o desempenho dos investidores privados (Barber e Odean, 2000, 2001, 2002; Seasholes e Zhu, 2005, 2007; Ivkovic, Sialm e Weisbenner, 2008; Kumar e Lee, 2006), em estudos sobre o desempenho de longo prazo das ações (Brav e Gompers, 1997; Fama, 1998; Mitchell e Stafford, 2000), na 
investigação sobre insider trading (Jaffe, 1974; Jeng, Metrick e Zeckhauser, 2003) e em estudos analisando o desempenho de fundos mútuos e de hedge fundos (Kacperczyk, Sialm, e Zheng, 2008; Fung, Hsieh, Naik e Ramadorai, 2008).

A grande vantagem e razão para a popularidade da abordagem é a sua capacidade para permitir a inferência estatística robusta quando a dependência transversal está presente. Ao agregar os retornos de uma secção transversal inteira em uma única carteira, o problema da dependência transversal entre os sujeitos individuais é eliminado (Lyon, Barber e Tsai, 1999).

\subsubsection{MODELAGEM DAS RESPOSTAS AO RETORNO DE AÇÕES}

A modelagem das respostas ao retorno de ações avalia se as informações contidas em uma medida estão associadas com o retorno de ações. O quadro para a definição do conteúdo de informação de uma medida decorre de estudo Ball e Brown (1968), o que estimulou uma extensa corrente de pesquisa voltada a examinar a relação entre a informação financeira (por exemplo, o lucro) e as informações do mercado de capitais.

Recentemente, este método foi estendido para avaliar o conteúdo das informações resultantes de medidas não financeiras. Estes estudos avaliam o "conteúdo incremental da informação" ou "a relevância de valor" das medidas não financeiras (ou seja, o grau com que o poder explicativo destas informações somadas à informação contábil explica os movimentos dos preços). Este desenvolvimento foi motivado pela observação de que os participantes do mercado de ações valorizam empresas com base não só na informação contábil (que não reflete totalmente o estado de ativos intangíveis e novas oportunidades de crescimento), mas também baseados em outras informações relevantes para o desempenho futuro. O modelo indica se os investidores percebem as informações sobre a atividade de marketing, tais como os gastos com publicidade, contribuindo para a projeção do fluxo de caixa futuro.

As comunicações de marketing devem refletir as informações que estão disponíveis para os participantes de mercado, uma vez que o mercado de ações reage à informação publicada. Estudos de Aaker e Jacobson (1994) abordaram os retornos decorrentes da percepção da qualidade e, também, os retornos das ações decorrentes da marca (Aaker, Jacobson, 2001).

\subsubsection{MODELO PERSISTENTE}

A questão da resposta do mercado a longo prazo está no cerne de todo estratégia de marketing que tenta criar uma vantagem competitiva sustentável para a empresa. Um desafio 
importante, porém, é que apenas os resultados de curto prazo das ações de marketing são facilmente observáveis. O Modelo persistente aborda o problema da quantificação das respostas do mercado a longo prazo, pela representação em uma medida de "impacto líquido de longo prazo", do conjunto de respostas do consumidor, feedback da empresa e respostas ao concorrente que surge na decorrência de uma ação de marketing.

Estes modelos utilizam a representação de um sistema em que cada equação acompanha o comportamento de um agente importante: o consumidor (equação da demanda), o gerente (equação da regra de decisão), a concorrência (equação reação competitiva) e o investidor (equação preço de ações). Fornece uma previsão, da base esperada para cada variável de desempenho e permite vários loops de feedback dinâmicos entre as variáveis de desempenho de marketing e ações. Pauwels et al. (2004) pesquisaram a avaliações de empresas de acordo com novos lançamentos de produtos e promoções de vendas, Joshi e Hanssens (2003, 2009, 2010) realizaram pesquisas sobre as consequências da publicidade nos retornos das empresas.

\subsection{MÉTRICAS FINANCEIRAS, VALOR DA EMPRESA E MÉTRICAS DE MARKETING}

A literatura de finanças distingue valor de informações entre fracas, moderadas e fortes para eficiência de mercado, sendo as ações de marketing via de regra, são classificadas como moderadas (Srinivasan, Hanssens, 2009). A teoria de finanças suporta o valor e relevância das estratégias de marketing devido aos seus efeitos no fluxo de caixa (Rao, Bharadwaj, 2008). O valor total das ações de uma empresa tem duas partes: retorno esperado e retorno anormal. Esses retornos dependem do tamanho do risco, valor do risco e fatores que compõem o risco de gerar fluxo de caixa futuro das empresas (Fama, French, 1992, 1996). O risco é formado pelo risco sistemático, que não depende apenas da empresa em avaliação, e o risco idiossincrático relacionado as ações da empresa no mercado (Hamilton, 1994). Luo e Bhattacharya (2009) estudaram a relação da performance social corporative, aliada às estratégias de marketing e o risco idiossincrático da firma.

Segundo Goyal e Santa-Clara (2003) 80\% do risco total depende do risco idiossincrático. Se marketing contribui para estabilizar volatilidade de caixa (ex: estratégias de fidelidade de clientes), incremento de fluxos de caixa anormais (ex: lançamento de novos produtos, melhoria da imagem da marca que gera manutenção ou incremento de preço de produtos e serviços), então as estratégias de marketing influenciam valor da firma por meio da diminuição do risco de fluxos de caixa futuros.

Assim, as estratégias de marketing precisam ser capturadas por meio de métricas que permitam investidores tomarem decisão em relação ao seu valor para o valor final da firma. As 
métricas de marketing são definidas em dois grupos - financeiras e não financeiras (Srivasan e Hanssens, 2009). Os exemplos de métricas financeiras de marketing se confundem com as métricas contábeis e econômicas das empresas que em geral capturam valores dentro do período contábil. Como exemplos de métricas não financeiras podem ser listadas Valor da Marca, Satisfação do Cliente Medidas do Cliente Qualidade do Produto.

\subsubsection{MÉTRICAS FINANCEIRAS}

Métricas financeiras devem ser definidas como o tipo de métricas onde é possível avaliar quantitativamente o resultado. Gaiardelli, Saccani e Songini (2007), utilizaram métricas financeiras para medir o desempenho da cadeia de suprimentos utilizando o modelo SCOR (Supply Chain Operations Reference), índices de rentabilidade (ROE, ROI, ROS) e o índice MSI (Media Sustentability Index). Métrica é um sistema de medida que quantifica a tendência, a dinâmica, ou alguma característica. Em praticamente todas as disciplinas, os profissionais usam métricas para explicar fenômenos, diagnosticar causas, compartilhar descobertas e projetar os resultados de eventos futuros. As métricas tornam possível comparar as observações e com isso facilitam a compreensão e os efeitos de uma determinada estratégia da empresa.

\subsubsection{VALOR DA EMPRESA E MÉTRICAS EM MARKETING}

\subsubsection{Valor da Empresa}

O valor da empresa, representada pela capitalização de mercado e o retorno das ações, de acordo com a HME, deve refletir toda a expectativa presente e futura de geração de valor desta empresa, consequentemente o valor de mercado desta empresa será dado pelo preço de mercado de sua ação multiplicado pelo número de ações emitidas.

De acordo com Srinivasan e Hanssens (2009), a melhor métrica para avaliar o valor para o acionista é o valor de mercado da empresa, o qual é calculado utilizando-se o preço da ação multiplicado pelo número de ações em circulação.

Os autores também sugerem a utilização de dois fatores para a operacionalização do valor da empresa: em primeiro lugar temos o valor contábil da empresa, que pode ser atingido utilizando o modelo de q de Tobin ou a relação market-to-book, que é a razão entre o valor de mercado de uma empresa e seu valor patrimonial (valor contábil) e expressa a valorização da empresa em relação aos seus dados contábeis, e em segundo lugar, a decisão de como tratar e incorporar o conceito de 
variação nos preços das ações conhecido como random walk (Fama, 1965). A avaliação do valor econômico de uma empresa já não se baseia unicamente nos fluxos físicos de matérias, mercadorias e produtos, mas nas expectativas de geração de valor representados pelos fluxos de dados, imagens e símbolos (Goldfinger, 2000).

A valorização da empresa, medida pelo preço de mercado da ação, decorrente das atividades de marketing, não têm recebido muita atenção de pesquisas. De forma geral, a hipótese do mercado eficiente, determina que o preço atual de uma ação considere imediatamente todas as informações conhecidas sobre a empresa, seja sobre lucros ou mesmos problemas futuros, os quais de uma forma ou de outra alteram as expectativas de lucro (Fama, French, 1992).

Ao contrário do comportamento de séries temporais típicos de vendas ao consumidor ou preços do produto, tem-se um componente permanente de flutuações nos preços de ações. No entanto, o mercado eficiente também reconhece que os investidores nem sempre prevêm corretamente e imediatamente os retornos futuros da empresa (Ball, Brown, 1968).

Os modelos de vetores autorregressivos (VAR), representam bem as respostas ao desempenho dinâmico e as interações entre o desempenho e as ações de marketing, que são variáveis endógenas (Dekimpe, Hanssens,1999). O modelo VAR calcula a linha de base de cada variável endógena e prevê seus valores futuros com base das interações dinâmicas de todas as variáveis conjuntamente endógenas (Pauwels et al., 2004).

Modelos de vetores autorregressivos têm sido utilizados no meio acadêmico envolvendo a área de marketing e a área de finanças (Pauwels et al., 2004). Na área de marketing estes modelos são utilizados na avaliação dos efeitos na performance da empresa causada pelas das ações no marketing mix (Dekimpe, Hanssens 1999; Bronnenberg, Mahajan,, Vanhonacker 2000; Nijs et al., 2001; Pauwels, Hanssens, Siddarth, 2002; Pauwels, 2004; Pauwels, Srinivasan, 2004; Srinivasan et al. 2004).

\subsubsection{Métricas de Marketing}

As métricas não financeiras não podem ser definidas em quantidade de dinheiro. Os Autores Greiling (2006) e Town (2000) compararam alguns estudos em seus artigos. De acordo com Zahay e Griffin (2010) as escalas utilizadas com os clientes não são tão rigorosas como nas métricas financeiras. Dentre as métricas de marketing, incluem-se o valor da marca, a satisfação do cliente, o ciclo de vida do cliente e a qualidade do produto (Kirmani, Wright, 1989; Greenyer, 2006). 
Yoo e Donthu (2001) verificaram empiricamente que o valor da marca é um construto multidimensional. Washburn e Plank (2002), Myers (2003), Kim et al (2003), Pappu et al. (2005), Villarejo-Ramos e Sánchez-Franco (2005) e Neto e Luce (2006), explicaram sua multidimensionalidade na perspectiva do consumidor. Barth et al. (1998) realizaram pesquisas onde concluíram que o retorno das ações são positivamente relacionadas com a valorização da marca. Simon e Sullivan (1993) perceberam que grande parte da valorização das empresas baseia-se no brand equity. Madden, Fehle e Fournier (2006) concluíram que marcas fortes proporcionam maiores retornos com um risco menor. Rao, Agarwal e Dahlhoff (2004) e Joshi e Hanssens (2003, 2010) relatam que o impacto da marca no valor da empresa é moderado pelo tipo de estratégia da marca, ou seja, se a marca é corporativa, empresa de marcas ou marca mista. Mizik e Jacobson (2007) pesquisaram as variações nos ativos da marca e as associações com as mudanças na avaliação do mercado financeiro. Cornwell, Roy e Steinard II (2001) exploraram o impacto das ações de patrocínio e seu impacto no valor da marca, pela perspectiva dos gestores.

A satisfação do cliente gera informações sobre as perspectiva dos fluxos de caixa da empresa (Anderson, Fornell, Mazvancheryl 2004; Gruca, Rego 2005), e os analistas sugerem negociação com ações baseados nas perspectivas dos fluxos de caixa da empresa (Chen, Matsumoto 2006; Womack, 1996). Satisfação leva a resultados positivos para com os clientes, tais como a fidelidade do cliente (Fornell et al. 2006), o boca a boca (Luo, 2009), e a disposição para pagar mais (Homburg, Koschate, Hoyer, 2005) que, por sua vez, aumenta os fluxos de caixa líquidos futuros (Aksoy et al., 2008;. Anderson, Fornell, Mazvancheryl, 2004). Ittner e Larcker (1998) pesquisaram o efeito o aumento da satisfação nos retornos anormais da empresa. Anderson, Fornell e Mazvancheryl (2004) concluíram que um aumento de $1 \%$ na satisfação do cliente provoca um aumento de $1.016 \%$ no q de Tobin. Gruca e Rego (2005) afirmam que o aumento na satisfação do cliente gera crescimento adicional no fluxo de caixa. Fornell et al. (2006) e Mittal et al. (2005) perceberam que clientes satisfeitos geram retornos positivos. Gupta e Zeithami (2006) descobriram forte relação entre satisfação do cliente, lucratividade da empresa e seu valor de mercado. Luo e Bhattacharya (2006) relacionaram responsabilidade social, satisfação do cliente e o valor de mercado da empresa.

Customer Lifetime Value (CLV) é o valor do relacionamento com o cliente para a empresa em termos monetários (Bell et al., 2002). De acordo com Rust, Zeithaml e Lemon (2001), o estudo da estrutura de valor para o cliente aponta os atributos relevantes para cada tipo de cliente, facilitando a formulação de estratégias e a construção de uma base de informação onde se possa projetar o valor financeiro do cliente durante a sua vida útil (Customer lifetime value - CLV). Rust, et al. (2004) propõem que as empresas devam buscar a melhoria em termos de valor do cliente, 
pois, estrategicamente, o esforço financeiro deve ser considerado um investimento e, portanto, uma forma de se atingir os objetivos financeiros.

O cliente é um ativo que as empresas e organizações devem mensurar, gerenciar e maximizar, assim como qualquer outro ativo (Blattberg, Getz, Thomas, 2001; Lemon, Rust, Zeithaml, 2001). De acordo com Venkatesan e Kumar (2004), o gerenciamento do valor do cliente fornece informações importantes para que a área de marketing desenvolva estratégias de longo prazo. Srinivasan e Hanssens (2009) afirmam que valor do cliente e valor de mercado estão intrinsecamente relacionados porque são duas versões baseadas no valor presente de um fluxo de caixa futuro esperado. Gupta, Lehmann e Stuart (2004) concluíram que valorizar clientes valoriza da empresa, pois isto acontece em três de cada cinco empresas pesquisadas. Concluíram, ainda, que a retenção é um caminho mais promissor para empresa, pois o seu efeito é muito mais significativo em relação à obtenção de novos clientes.

Aaker e Jacobson (1994) e Mizik e Jacobson (2004) concluíram que mudanças na qualidade percebida estão associadas ao retorno das ações. Investidores percebem a qualidade, fornecendo informações úteis sobre as perspectivas futuras da empresa. Quanto à qualidade do produto, sua relação com a avaliação de mercado é uma área relativamente nova em termos de pesquisa, visto que existem diferentes definições de qualidade (Mitra, Golder, 2006). Dentre os poucos estudos sob esta perspectiva, Kirmani e Wright (1989) analisaram o impacto da percepção dos gastos com publicidade e seu impacto na expectativa de qualidade do produto. Em resumo, é preciso mais do que apenas a introdução de novos produtos para melhorar o desempenho das ações. Melhorias na avaliação do consumidor em termos de qualidade percebida, especialmente para novos produtos, estão significativamente relacionadas com o valor da empresa (Srinivasan, Hanssens, 2009).

\subsection{RETORNOS ESPERADOS E ANORMAIS}

Um dos pressupostos da Hipótese do Mercado Eficiente é que os agentes são capazes de processar de maneira ótima as informações disponíveis, visto que haveria disponibilidade de informações de maneira igualitária para todos e, portanto, não se justifica dispender tempo e recursos para obter informações públicas, objetivando retornos acima do retorno médio de mercado, pois estas informações já estariam incorporadas nos preços (Fama, 1970).

Kahneman e Tversky (1979) afirmam que os indivíduos tendem a dar peso excessivo a eventos considerados relativamente certos comparados com os meramente prováveis. Soma-se o fato do indivíduo ter capacidade limitada para processar informações e os custos para se identificar 
a decisão ótima implicarem em atalhos mentais para simplificar a decisão. Dentro do contexto da discussão entre a Hipótese do Mercado Eficiente e Finanças Comportamentais, tem-se alguns pontos comuns, como afirma De Bondt e Thaler (1985) sobre a disponibilidade de informações diretamente correlacionadas com a previsibilidade dos preços.

\subsection{NOVOS ESTUDOS}

Estudos posteriores ao de Srinivasan e Hanssens (2009) abordaram diferentes perspectivas entre finanças e marketing, mais particularmente relacionando fluxo de caixa e risco não sistemático.

Kimbrough e McAlister (2009) desenvolveram amplo estudo sobre a relação entre o valor da empresa e as estratégias de marketing, tecendo apontamentos a respeito das métricas, dos métodos, dos principais resultados empíricos, bem como desenvolveram direções para pesquisas futuras. Luo, Xueming e Pieter de Jong (2012) apontam que ainda existem desafios na concernentes às relações entre publicidade e mercado financeiro, representado pelo retorno das ações, o que pode levar a conclusões enganosas.

Críticos argumentam que a publicidade está muito longe do valor das ações de uma empresa e que deve haver algo no meio para explicar melhor. Pesquisas anteriores incidem mais sobre se os retornos relacionados à publicidade são significativos, porem desconhecem mecanismos de contabilização subjacente que apontem a presença ou ausência dos referidos retornos decorrentes de publicidade. Muitos trabalhos foram desenvolvidos. Joshi e Hanssens (2009, 2010), por exemplo, perceberam que a publicidade aumenta os retornos para algumas empresas, mas não todas. Shah et al. (2008) avaliaram mais de 150 estudos empíricos e encontraram resultados inconclusivos. Chemmanur e Yan analisaram questões pertinentes ao anúncio de produtos e sua relação com o valor da marca (2009a), bem como sua relação com o valor das ações (2009b).

Luo, Xueming e Pieter de Jong (2012), sugerem que algumas das inconsistências na literatura podem surgir porque estudos anteriores não têm os processos subjacentes examinados adequadamente de forma a conectar a publicidade com o valor da empresa. Ou seja, a literatura tem geralmente dado pouca atenção aos mecanismos intermediários através dos quais a publicidade pode criar valor da empresa (Kimbrough, McAlister, 2009).

Diante do impasse sobre a articulação entre finanças e marketing, surgiram outros trabalhos. Sood e Tellis (2009) sustenta que o impacto da publicidade sobre as vendas e o desempenho financeiro é altamente variável. Já Joshi e Hanssens (2010) examinaram o papel das vendas e dos lucros na relação do impacto da publicidade sobre o valor da empresa. Osinga et al. (2011) estimaram simultaneamente, os efeitos do marketing em todos os três componentes do valor para o 
acionista. O efeito de novos produtos no valor da empresa também foi investigado, pela perspectiva da influência da publicidade e de pesquisa e desenvolvimento (R\&D) sobre os retornos das ações (Joshi, Hanssens, 2010). Outros trabalhos, ainda, relacionaram a publicidade com o risco sistemático da empresa, como Fischer, Shin, e Hanssens (2009) que afirmaram que a publicidade reduz a volatilidade do fluxo de caixa e, portanto, o risco sistemático. Além disso, Luo, Homburg e Wieseke (2010) evidenciaram os resultados da satisfação do consumidor e das recomendações dos analistas de mercado no valor da firma.

\section{CONSIDERAÇÕES FINAIS}

Mesmo após o estudo de Srinivasan e Hanssens (2009) e a atualização presente neste artigo, alguns gaps ainda persistem. Tais questões de pesquisa foram discutidas em termos de seus específicos interesses e podem gerar diferentes linhas de estudo articulando estratégias de marketing e finanças no Brasil.

Gaps que discutem o valor das estratégias de marketing nas métricas financeiras do valor da empresa, e a relação das estratégias de marketing e volatilidade dos fluxos de caixa futuros geraram as duas questões a seguir

1. Qual é a melhor abordagem para quantificar o valor dos intangíveis (por exemplo, marcas, propriedade intelectual) e avaliar o seu impacto sobre os fluxos de caixa, crescimento e risco?

2. Compreender a componente volatilidade do valor da empresa: Em particular, se maiores níveis de brand equity, o patrimônio do cliente e variedade de produtos reduz a vulnerabilidade das empresas para incursões competitivas, reduzindo assim o risco e a volatilidade dos fluxos de caixa? Será que isso resulta em perfis de risco favoráveis (Ps inferiores)? Além disso, qual é a relação entre a volatilidade dos fluxos de caixa (ou volatilidade nos ganhos) e risco de mercado sistemático da empresa (ou seja, $\beta$ )? (Srinivasan, Hanssens, 2009, p. 308)

As medidas utilizadas em marketing e finanças em geral acompanham o ano contábil, o que continua gerando problemas em capturar o valor agregado pelas estratégias de marketing. Nesse sentido as questões 3 e 4 podem motivar a outra linha de pesquisa em marketing e finanças.

3. Tendo em conta que os benefícios da estratégia de branding e marketing são normalmente materializado ao longo de vários períodos, essas medidas de retorno sobre o investimento em marketing não são míopes?

4. Lidar com as pressões de receitas a curto prazo: Até o momento, a evidência empírica suporta a noção de que o mercado de ações não é míope. Assim, as empresas que se dedicam a gastos com marketing estratégico eficaz deve sentir-se segura em suas ações. No entanto, muitos executivos estão preocupados com as suas métricas de desempenho 
trimestrais, o que motiva algumas de suas ações. Como podem estes dois comportamentos aparentemente contraditórios se reconciliam? (Srinivasan, Hanssens, 2009, p. 308)

Questões comportamentais relacionadas ás reações dos investidores incentivam a investigação sobre de que forma investidores reagem as ações de marketing divulgadas no mercado. Ainda são pouco discutidas as possíveis interpretações de investidores com diferentes níveis de especialização referente a eficiência das estratégias de marketing.

5. Identificar as condições na qual a reação do investidor é precisa e quanto tempo leva para acontecer esta reação por parte do investidor. Dada a evidência mista sobre a qualidade da reação dos investidores, é importante entender quando ocorrem os desvios e como eles podem ser corrigidos. (Srinivasan, Hanssens, 2009, p. 308)

Em países como os Estados Unidos, analistas especializados podem influenciar a interpretação e valoração das estratégias de marketing, mas, no Brasil - onde esse agente econômico não é comum - abre-se a questão para buscar o entendimento se existe algum meio de comunicação interveniente entre o valor percebido das estratégias de marketing das empresas e a valoração dessas estratégias pelos investidores.

6. Como as interpretações de atividades de marketing pelos analistas de mídias especializadas afetam os retornos de ações? Existe uma diferença no comportamento dos retornos das ações de empresas que são mais visadas pelas mídias especializadas versus aqueles que não o são? Quanto tempo leva para os investidores perceberem os vieses?

Questões como estas ainda persistem e estudos futuros devem centrar esforços na investigação empírica nos mercados nacional e internacional. 


\section{REFERÊNCIAS}

Aaker, D. A.; R. Jacobson (1994). Study shows brand-building pays off for stockholders. Advertising Age 65(30),.

Aaker, D. A., R. Jacobson (2001). The Value Relevance of Brand Attitude in High-Technology Markets. Journal of Marketing Research, 31 (May), p. 191-201.

Aksoy, L.; Cooil, B.; Groening, C.; Keiningham, T. L.; Yalçin, A. (2008). The Long-Term Stock Market Valuation of Customer Satisfaction. Journal of Marketing, 72 (July), 105-122.

Ambler, T. (2003) Marketing and the Bottom Line. London: Financial Times/Prentice Hall.

Anderson, E. W.; Fornell, C.; Mazvancheryl, S. K. (2004). Customer Satisfaction and Shareholder Value. Journal of Marketing, 68 (October), 172-85.

Anderson, P. F. (1982). Marketing, Strategic Planning and the Theory of the Firm. Journal of Marketing, 46 (Spring), 15-26.

Ball, R.; Brown, B. (1968). An Empirical Evaluation of Accounting Income Numbers. Journal of Accounting Research, 6 (Autumn), 159-78.

Barber, B. M.; Odean, T. (2000). Trading is hazardous to your wealth: The common stock investment performance of individual investors. Journal of Finance, 55, 773-806.

(2001). Boys will be boys: Gender, overconfidence, and common stock investment, Quarterly Journal of Economics, 116, 261-292.

(2002) Online investors: Do the slow die first? Review of Financial Studies, 15, 455487.

Barth, M. E.; Clement, M.; Foster, G.; Kasznik, R. (1998) Brand Values and Capital Market Valuation. Review of Accounting Studies, 3, 41-68.

Braga, C., Costa Jr., N. C .A.; Mescolin A. (1997). Risco e retorno das value e growth stocks no mercado de capitais brasileiro. In: XXI EnANPAD - Encontro Anual da ANPAD, 1997. Anais. Rio de Janeiro: ANPAD.

Braga, C; Leal, R. (2000) Ações de valor e de crescimento nos anos 90. Relatório Coppead, v.330, 16p, Rio de Janeiro, (Agosto). 
Brandão, M. M.; Oliveira, F. R.; Goldner, F.; Gollner, E. S. (2006). Marketing e performance no setor bancário brasileiro. In: X Congresso USP de Controladoria e Contabilidade, São Paulo, Anais. São Paulo: USP, p. 1-15..

Brav, A.; Gompers, P. (1997) Myth or reality? The long-run underperformance of initial public offerings: Evidence from venture and non-venture-backed companies, Journal of Finance, 52, 1791-1821.

Bronnenberg, B. J., Mahajan, V.; Vanhonacker, W. (2000). The Emergence of Market Structure in New Repeat- Purchase Categories: A Dynamic Approach and an Empirical Application. Journal of Marketing Research, 37 (February), 16-31.

Byzalov, D.; Shachar, R. (2004). The risk reduction role of advertising. Quant Mark Econ, 2(4), 283-320.

Capon, N.; Farley, J. U.; Hoenig, S. (1990) Determinants of Financial Performance: A MetaAnalysis. Management Science, 36 (10), p. 1143-1159.

Cardoso, N. (2006). Santander ad-hoc quantitative - Fama \& French visit Brazil. Latin America Equity Research, quantitative report, july.

Chaney, P. K., Devinney, T. M.; Winer, R. S. (1991). The impact of new product introduction on the marketvalue of firms, Journal of Business, 64(4), 573-610.

CMO Council Measures and Metrics: Assessing Marketing's Value and Impact, Palo Alto, California: The CMO Council, 2004.

Cornwell, B. T.; Roy, D. P.; Steinard II, E. A. (2001). Exploring Manager's Perceptions of the Impact of Sponsorship on Brand Equity. Journal of Advertising, 30(2), p. 41-51.

Costa Jr.; Neves, M. (2000). Variáveis fundamentalistas e o retorno das ações. Revista Brasileira de Economia, v. 54, n. 1, p. 123-137, jan/fev/.mar.

Chemmanur, T.; Yan, A. (2009a) Product market advertising and new equity issues. J Financ Econ, 92(1), 40-65.

(2009b). Advertising, Attention and Stock Returns. Working paper, Boston College.

Day, G. S.; Fahey, L. (1988). Valuing Marketing Strategies. Journal of Marketing, 52 (July), 45-57.

De Bondt, W.; Thaler, R. (1985) Does the stock market overreact?. Journal of Finance. 40(3), p. 793-808. 
Dekimpe, M. G.; Hanssens, D. M. (1995). The Persistence of Marketing Effects on Sales. Marketing Science, 14 (1), 1-21.

Doyle, P. (2000). Value-Based Marketing: Marketing Strategies for Corporate Growth and Shareholder Value. Chichester, UK: John Wiley \& Sons.

Erickson, G.; Jacobson, R. (1992). Gaining Comparative Advantage Through Discretionary Expenditures: The Returns to R\&D and Advertising. Management Science, 38 (September), $1264-1279$.

Estellita, M.; Carvalho, F. A.; Lima, A. V. (2006). Marketing e desempenho nas empresas brasileiras: um estudo empírico usando dados de balanços para o período 1998-2003. In: XXX ENANPAD, Salvador, Anais. Salvador: ANPAD, p. 1-16. 1 CD-ROM.

Fama, E. F. (1965). Random walks in stock market prices. Financial Analysts Journal. Spt-oct, p. 55-59.

(1970). Efficient Capital Markets: a review of theory and empirical work. The Journal of Finance. V. 25, 2, May, 383-417.

(1998). Market efficiency, long-term returns and behavioral finance, Journal of Financial Economics, 49, 283-307.

Fama, E. F.; French, K. R. The cross-section of expected stock returns. The Journal of Finance, v.47, p.427-465, 1992.

(1993). Common risk factors in the returns on stocks and bonds. Journal of Financial Economics, v. 33, p. 3-56.

(1995a). The CAPM is wanted dead or alive. Working Paper, Chicago University.

(1995b). Size and book-to-market factors in earnings and returns. The Journal of Finance, v. 50, p. 131-55.

55-184.

(1996). Multifactor Explanations of Asset Pricing Anomalies. Journal of Finance, 51,

(1998). Value versus Growth: The International Evidence, Journal of Finance, 53, 6, p. 1975-1998.

Fehle, F.; Tsyplakov, S.; Zdorovtsov, V. (2005). Can Companies Influence Investor Behavior Through Advertising? Super Bowl Commercials and Stock Returns. European Financial Management, 11 (5), 625-647. 
Fornell, C.; Mithas, S.; Morgeso, F.; Krishnan, M. S. (2006). Customer Satisfaction and Stock Prices: High Returns, Low Risk. Journal of Marketing, 70 (January), 3-14.

Fortunato, G.; Ness, W.; Motta, P. C. (2009) Interação dos dispêndios de publicidade com ciclos econômicos e valor da empresa: análise empírica das empresas norte-americanas. In: XXXIII EnANPAD, São Paulo, Anais. São Paulo: ANPAD, p. 1-16.

Freundt, V. L. M. A. (2012). Métricas de avaliação de comunicação de marketing offline e online: um estudo sobre o setor de bancos. Tese de Doutorado. Universidade de São Paulo. São Paulo.

Frieder, L.; Subrahmanyam, A. (2005). Brand Perceptions and the Market for Common Stock. Journal of Financial and Quantitative Analysis, 40 (1), 57-85.

Fung, W.; Hsieh, D. A.; Naik, N. Y.; Ramadorai, T. (2008). Hedge funds: Performance, risk, and capital formation, Journal of Finance, 63, 1777-1803.

Gaiardelli, P., Saccani, N.; Songini, L. (2007). Performance measurement of the after-sales service network: Evidence from the automotive industry. Computers in Industry, Vol. 58, 7, pp. 698708.

Goldfinger, C. (2000). Intangible economy and financial markets. Communications \& Strategies, n. 40, 4th quarter, p. 59.

Goyal, A.; Santa-Clara, P. (2003) Idiosyncratic Risk Matters! Journal of Finance, 58 (3), 975-1007.

Granger, C.; Newbold, P. (1986). Forecasting Economic Time Series. Orlando, FL: Academic Press.

Greenyer, A. (2006). Measurable marketing: a review of development in marketing's measurability. Journal of Business \& Industrial Marketing, Vol. 21, 4, pp. 239-242.

Greiling, D. (2006). Performance measurement: A remedy for increasing the efficiency of public services? International Journal of Productivity and Performance Management, 55(6), 448-465.

Gruca, T. S.; Rego, L. L. (2005). Customer Satisfaction, Cash Flow, and Shareholder Value", Journal of Marketing, 69 (July), 115-30.

Grullon, G.; Kanatas, G.; Weston, J. P. (2004) Advertising, Breadth of Ownership and Liquidity. Review of Financial Studies, 17 (2), 439-61.

Gupta, S.; Zeithaml, V. (2006). Customer Metrics and Their Impact on Financial Performance," Marketing Science, 25 (6), 687-717. 
Gupta, S.; Lehmann, D. R.; Stuart, J. A. (2004). Valuing Customers. Journal of Marketing Research, 41 (February), 7-18.

Hazzan, S. (1991) Desempenho de Ações da Bolsa de Valores de São Paulo e sua relação com o índice preço-lucro. Tese de Doutorado, São Paulo, EAESP/FGV, 263p.

Homburg, C.; Koschate, N. Hoyer, W. D. (2005) Do Satisfied Customers Really Pay More? A Study of the Relationship Between Customer Satisfaction and Willingness to Pay. Journal of Marketing, 69 (April), 84-96.

Hyman, M.; Mathur, K. (2005). Retrospective and Prospective Views on the Marketing Finance Interface. Journal of the Academy of Marketing Science. 33, p. 373-381.

Ittner, C.; Larcker, D. Are Non-Financial Measures Leading Indicators of Financial Performance? An Analysis of Customer Satisfaction. Journal of Accounting Research, 36 (3, Supplement), 135, 1998.

Ivkovic, Z.; Sialm, C.; Weisbenner, S. (2008). Portfolio concentration and the performance of individual investors, Journal of Financial and Quantitative Analysis 43, 613-656.

Jaffe, J. F. (1974). Special information and insider trading, Journal of Business 47, 410-428.

Jeng, L. A., Metrick, A.; Zeckhauser, R. (2003) Estimating the returns to insider trading: A performance-evaluation perspective, Review of Economics and Statistics 85, 453-471.

Joshi, A.; Hanssens, D. M. (2003). Advertising Spending and Market Capitalization, Working Paper, (UCLA Anderson Graduate School of Management), August.

(2009). Movie advertising and the stock market valuation of studios: a case of Great expectations? Marketing Science, 8(2), 239-250, 2009.

(2010). The Direct and Indirect Effects of Advertising Spending on Firm Value. Journal of Marketing, 74 (January), 20-33.

Kacperczyk, M.; Sialm, C.; Zheng, L. (2008). Unobserved actions of mutual funds, Review of Financial Studies 21, 2379-2416.

Kaldor, N. (1950). The economic aspects of advertising. Review of Economic Studies, 18, p. 1-27.

Kahneman, D; Tversky, A. (1979). Prospect Theory: An analysis of Decision under risk. Econometrica. Vol. 47, p. 263-291. 
Kim, H.; Kim, W. G.; An, J. A. (2003) The effect of consumer-based brand equity on firm's financial performance. Journal of Consumer Marketing, v. 20, n. 4, p. 335-351.

Kimbrough, M.; McAlister, L. (2009). Commentaries and rejoinder to Marketing and firm value: metrics, methods, findings, and future directions. Journal of Marketing Research, 46(3), 313329.

Kirmani, A.; Wright, P. (1989). Money talks: perceived advertising expense and expected product quality. Journal of Consumer Research, 16, 344-353.

Knowles, J. (2003). Value-Based Brand Measurement and Management. Interactive Marketing 5 (July/September): 40-50.

Koslow, S.; Sasser, S.; Riordan, E. (2006). Do marketers get the advertising they need or the advertising they deserve? Journal of Advertising, 35(3), 81-101.

Kremer, S. T. M., Tammo, H. A.; Bijmolt, P.; Leeflang, S. H.; Wieringa, J. E. (2008). Generalizations on Pharmaceutical Marketing Effectiveness. International Journal of Research in Marketing, 25 (4), 234-46.

Kumar, A.; Lee, C. M. C.; (2006). Retail investor sentiment and return comovements. Journal of Finance 61, 2451-2486.

Leone, R. (1995). Generalizing what is known of temporal aggregation and advertising carryover. Marketing Scince, 14(3), G141-G150.

Lane, V.; Jacobson, R. (1995). Stock market reactions to brand extensions announcements: the effect of brand attitude and familiarity. Journal of Marketing, 59(1),63-77.

Lehmann, D. R. (2004). Linking Marketing to Financial Performance and Firm Value. Journal of Marketing, 68 (October),73-75.

Luo, X.; Donthu, N. (2006). Marketing's Credibility: A Longitudinal Investigation of Marketing Communication Productivity and Shareholder Value. Journal of Marketing: October, Vol. 70, No. 4, pp. 70-91.

Luo, X. Bhattacharya, C. B. (2006). Corporate Social Responsibility, Customer Satisfaction, and Market Value. Journal of Marketing, 70 (July), 1-18.

(2009). The Debate over Doing Good: Corporate Social Performance, Strategic Marketing Levers, and Firm-Idiosyncratic Risk. Journal of Marketing, 73 (November), 198-213. 
Luo, X.; De Jong, P. (2012). Does Advertising Spending Really Work? The Intermediate Role of Analysts in the Impact of Advertising on Firm Value. Journal of the Academy of Marketing Science, 40, 605-624.

Luo, X. (2009). Quantifying the Long-Term Impact of Negative Word of Mouth on Cash Flows and Stock Prices," Marketing Science, 28 (1), 148-165.

Luo X.; Homburg, C.; Wieseke, J. (2010). Customer satisfaction, analyst stock recommendations, and firm value. Journal of Marketing Research, 47, 6, 1041-1058.

Lyon, J. D., Barber, B. M.; Tsai, C. (1999). Improved methods for tests of long-run abnormal stock returns, Journal of Finance 54, 165-201.

Madden, T. J., Fehle, F. Fournier, S. (2006). Brands Matter: An Empirical Demonstration of the Creation of Shareholder Value through Branding. Journal of the Academy of Marketing Science, 34(2), 224-235.

Manchanda, P.; Wittink, D. R.; Ching, A.; Cleanthous, P.; Xiaojing, M. D.; Dong, J. et al. (2005). Understanding Firm, Physician and Consumer Choice Behavior in the Pharmaceutical Industry. Marketing Letters, 16 (3-4), 293-308.

Mandelker, Gershon. (1974). Risk and return: The case of merging firms. Journal of Financial Economics, December, 1 (4), 303-335.

Martins, M. A. M. (2011). Os gastos com propaganda e o valor ao acionista. 175f. Dissertação (Mestrado em Administração). Universidade Federal do Rio Grande do Sul, Porto Alegre.

McAlister, L.; Srinivasan, R.; Kim, M. C. (2007). Advertising, Research and Development, and Systematic Risk of the Firm. Journal of Marketing, 71 (January), 35-48.

Mitchell, M. L.; Stafford, E. (2000). Managerial decisions and long-term stock price performance, Journal of Business 73, 287-329.

Mittal, V.; Anderson, E. W.; Sayrak, A.; Tadikamalla, P. (2005). Dual Emphasis and the LongTerm Financial Impact of Customer Satisfaction. Marketing Science, 24 (4), 544-55.

Mizik, N.; Jacobson, R. (2003). Trading Off Between Value Creation and Value Appropriation: The Financial Implications of Shifts in Strategic Emphasis. Journal of Marketing, 67 (January), 6376.

Myers, C. A. (2003). Managing brand equity: a look at the impact of attributes. Journal of Product and Brand Management, v. 12, n. 1, p. 39-51. 
Neto, A. V.; Luce, F. B. (2006). Mensuração de brand equity baseado no consumidor: avaliação de escala multidimensional. In: II EMA - Encontro de Marketing da ANPAD, maio, Rio de Janeiro, Brasil.

Nijs, V.; Dekimpe, M.; Steenkamp, J. E. M.; Hanssens, D. M. (2001). The Category Demand Effects of Price Promotions. Marketing Science, 20 (1), 1-22.

Osinga, E. C.; Leeflang, P. S. H.; Srinivasan, S.; Wieringa, J. E. (2010). Why do firms invest in consumer advertising with limited sales response? A shareholder perspective. Journal of Marketing, 75(1), 109-124.

Pappu, R.; Quester, P. G.; Cooksey, R. W. (2005) Consumer-based brand equity: improving the measurement - empirical evidence. Journal of Product and Brand Management, v. 14, n. 3, p. 143-154.

Pauwels, K.; Srinivasan, S. (2004). Who Benefits from Store Brand Entry? Marketing Science, 23, 3, 364-390.

Pauwels, K.; Hanssens, D. M.; Siddarth, S. (2002) The Long-Term Effects of Price Promotions on Category Incidence, Brand Choice, and Purchase Quantity. Journal of Marketing Research, 34 (November), 421-39.

Pauwels, K.; Silva-Risso, J.; Srinivasan, S.; Hanssens, D. M. (2004) New Products, Sales Promotions, and Firm Value: The Case of the Automobile Industry. Journal of Marketing, 58, October, 142-156.

Ramesh K. S. R.; Bharadwaj, N. (2008) Marketing Initiatives, Expected Cash Flows, and Shareholders' Wealth. Journal of Marketing, 72, 16-26.

Rao, V. R., Agarwal, M. K.; Dahlhoff, D. (2004). How Is Manifest Branding Strategy Related to the Intangible Value of a Corporation? Journal of Marketing, 68(4), 126-141.

Rao, K. S.; Bharadwaj, N. (2008). Mrkating initiatives, expected cash flows and shareholders' wealth. Journal of Marketing, 72, January, 16-26.

Rust, R. T.; Lemon, K. M.; Zeithalm, V. A. (2004a). Return on marketing: Using customer equity to focus marketing strategy. Journal of Marketing, Vol. 68, No. 1 (January), pp. 109-127.

Rust, R. T.; Ambler, T.; Carpenter, G. S.; Kumar, V.; Srivastava, R. K. (2004b). Measuring Marketing Productivity: Current Knowledge and Future Directions. Journal of Marketing, 68 (October), 76-89.

Rust, R. T.; Zeithaml, V.; Lemon, K. N. (2001). O valor do cliente. Porto Alegre: Bookman. 
Seasholes, M. S.; Zhu, N. (2005). Is there information in the local portfolio choices of individuals? Working Paper, University of California Berkeley.

(2007) Individual investors and information diffusion, Working Paper, University of California Berkeley.

Sheth, J. N.; Sisodia, R. S. (2001). High performance marketing: Marketing must be a leader for change across the corporation. Marketing Management, Vol. 10, No. 3, pp. 18-23.

Simon, C. J.; Sullivan, M. W. (1993). The Measurement and Determinants of Brand Equity: A Financial Approach. Marketing Science, 12 (1), 28-52.

Sood, A.; Tellis, G. J. (2009). Do Innovations Really Pay Off? Total Stock Market Returns to Innovation. Marketing Science, 28 (3), 442-56.

Srinivasan, S.; Hanssens, D. M. (2009). Marketing and Firm Value: Metrics, Methods, Findings, and Future Directions. Journal of Marketing Research, 46 (June), 293-312.

Srinivasan, S; Pauwels, K.; Hanssens, D. M.; Dekimpe, M. (2004). Do Promotions Benefit Retailers, Manufacturers, or Both? Management Science, 50 (5) 617-29.

Srivastava, R. K.; Shervani, T. A.; Fahey, L. (1998). Market-Based Assets and Shareholder Value: A Framework for Analysis. Journal of Marketing, Vol. 62 (January), p. 2-18.

Szymanski, D. M., Bharadwaj, S. G.; Varadarajan, P. R. (1993). An Analysis of the Market ShareProfitability Relationship. Journal of Marketing, 57(July), 1-18.

Tellis, G. (2009). Generalizations about advertising effectiveness in markets. Journal of Advertising Research, 49(2), 240-245.

Town, J. S. (2000). Performance or measurement? In Performance Measurement and Metrics, Vol. 1 Iss: 1, pp. 43-54.

Venkatesan, R.; Kumar, V. (2004). A Customer Lifetime Value Framework for Customer Selection and Resource Allocation Strategy. Journal of Marketing, 68 (October): 106-125.

Villarejo-Ramos, A. F.; Sánchez-Franco, M. J. (2005). The impact of marketing communication and price promotion on brand equity. Brand Management, v. 12, n. 6, p. 431-444.

Washburn, J. H.; Plank, R. E. (2002). Measuring Brand Equity: An Evaluation of a Consumerbased Brand Equity Scale. Journal of Marketing Theory and Practice, 10 (1): 46-62. 
Woodburn, D. (2004). Engaging marketing in performance measurement. Measuring Business Excellence, Vol. 8, No. 4, pp. 63-72.

Wosinska, M. (2005). Direct-to-Consumer Advertising and Drug Therapy Compliance. Journal of Marketing Research, 42 (August), 323-32.

Yoo, B.; Donthu, N. (2001). Developing and validating a multidimensional consumer-based brand equity scale. Journal of Business Research, v. 52, p. 1-14.

Zahay, D. E.; Griffin, A. (2010). Marketing Strategy Selection, Marketing Metrics and Firm Performance. Journal of Business and Industrial Marketing, 25:2, 84-93 (lead article).

Recebido: 24/08/2013

Aprovado: 15/10/2013 